\title{
Active Galaxies and Cluster Gas
}

\author{
BY A.C FABIAN \\ Institute of Astronomy, Madingley Road, Cambridge CB3 OHA, UK
}

Two lines of evidence indicate that active galaxies, principally radio galaxies, have heated the diffuse hot gas in clusters. The first is the general need for additional heating to explain the steepness of the X-ray luminosity-temperature relation in clusters, the second is to solve the cooling flow problem in cluster cores. The inner core of many clusters is radiating energy as X-rays on a timescale much shorter than its likely age. Although the temperature in this region drops by a factor of about 3 from that of the surrounding gas, little evidence is found for gas much cooler than that. Some form of heating appears to be taking place, probably by energy transported outward from the central accreting black hole or radio source. How that energy heats the gas depends on poorly understood transport properties (conductivity and viscosity) of the intracluster medium. Viscous heating is discussed as a possibility. Such heating processes have consequences for the truncation of the luminosity function of massive galaxies.

Keywords: X-rays; active galaxies; clusters; cooling flows

\section{Introduction}

Clusters of galaxies are luminous X-ray sources, with X-ray luminosities $L_{\mathrm{X}} \sim$ $10^{43}-10^{46} \mathrm{erg} \mathrm{s}^{-1}$. The emission is predominantly thermal bremsstrahlung from highly ionized hydrogen and helium in the intracluster medium (ICM) at temperatures $T \sim 10^{7}-1.5 \times 10^{8} \mathrm{~K}$. Line emission, particularly from iron, is also present showing that most of the gas has a mean metallicity of about 0.3 Solar. The total mass of the intracluster medium is about one sixth of the total cluster mass, and the stars in all the member galaxies have about one sixth of the mass of the hot gas. Most of the total mass of a cluster is due to dark matter.

Simple gravitational collapse models for clusters scale such that the $L_{\mathrm{X}} \propto T^{2}$ whereas the data are better fit by $L_{\mathrm{X}} \propto T^{3}$ or even steeper at low ICM temperatures. This can be explained by additional heating of the ICM to a level of $1-3 \mathrm{keV}$ per particle. Supernovae are unable to supply this much heat and active galactic nuclei (AGN) within a cluster, or its consitutent subclusters at an earlier epoch, seem the most likely heat source on energetic grounds (Valageas \& Silk 1999; Wu, Fabian \& Nulsen 2000).

The X-ray emission in the cores of many clusters is sharply peaked. The radiative cooling time of the gas within $50 \mathrm{kpc}$ of the centre is shorter than the likely age of the cluster. The temperature drops smoothly there by a factor of two to three from that of the outer gas. Although it might seem that a cooling flow should be operating there with gas cooling out of the intracluster medium, spectra from XMM-Newton and Chandra show that radiative cooling is much reduced and some form of distributed heating is taking place. 
This last point is of considerable importance for understanding the gaseous part of galaxy formation, much of which proceeds by radiative cooling of hot gas in dark matter potential wells. The cooling in galaxies predominately occurs in the extreme and far UV and so is not readily observable, but is directly observable in clusters. Whatever is stemming the cooling in clusters may be determining the upper mass cutoff for galaxies.

The AGN in the central galaxy is a viable culprit for heating the core region of clusters. AGN may therefore play a significant role in determining the properties of the ICM, both generally for the whole cluster and specifically for the inner region.

\section{Evidence for AGN heating of clusters at moderate to high redshifts}

Massive galaxies host massive black holes built by accretion over the redshift range $2-4$. Many of them plausibly were powerful radio galaxies at some stage, injecting vast amounts of energy into surrounding gas. Integrating the radio galaxy luminosity function over time leads to a possible comoving energy input of $\sim$ $10^{57} \mathrm{erg} \mathrm{Mpc}^{-3}$ (Inoue \& Sasaki 2001). This can preheat the ICM to 1-2 keV per particle, as required to account for the observed $L_{\mathrm{X}} \propto T^{3}$ rather than $T^{2}$ as expected from gravity alone.

Studying this AGN-cluster connection further is not easy. We need to know the kinetic power of jets and their lifetime. Radio observations of course show us that such sources exist but synchrotron and Compton losses on the electrons mean that the observable lifetime is short. One recent development which should help is the detection with Chandra of extended X-ray emission from high redshift radio galaxies. The emission is likely inverse Compton scattering of Cosmic Microwave Background (CMB) photons by electrons with Lorentz factor $\gamma \sim 1000$. The steep increase in the energy density of the CMB with redshift, as $(1+z)^{4}$, partially compensates for the large distance to the sources, so making them observable. By $z \sim 2, L_{\mathrm{X}}$ for a given electron population is boosted by a factor of nearly 100 compared with the present epoch.

Examples of such sources are 3C294 $(z=1.786$, Fabian et al 2003c), 3C9 $(z=2.01$, Fabian, Celotti \& Johnstone 2003), PKS 1138-262 (Carilli et al 2002), $4 \mathrm{C} 41.17(z=3.8$, Scharf et al 2003) and GB1508+5714 $(z=4.3$, Yuan et al 2003; Siemiginowska et al 2003). As discussed by Celotti \& Fabian (2004), similar sources should emerge from further Chandra observations of distant radio galaxies. They should dominate the extended sources in the X-ray Sky at $z>2$ and $L_{\mathrm{X}}>10^{44} \mathrm{erg} \mathrm{s}^{-1}$ (Fig. 1). Since the X-ray emission involves lower energy electrons than typically involved in the observed radio emission, a source should be detectable longer after an outburst in the X-ray band than in the radio. (Note that the X-ray extent of $3 \mathrm{C} 294$ exceeds the radio emission by $\sim 50 \mathrm{kpc}$ or more to the NW and SE.) This means that some unidentified faint extended X-ray sources (Bauer et al 2002) could be due to inverse Compton emission from faded radio galaxies.

Article submitted to Royal Society 


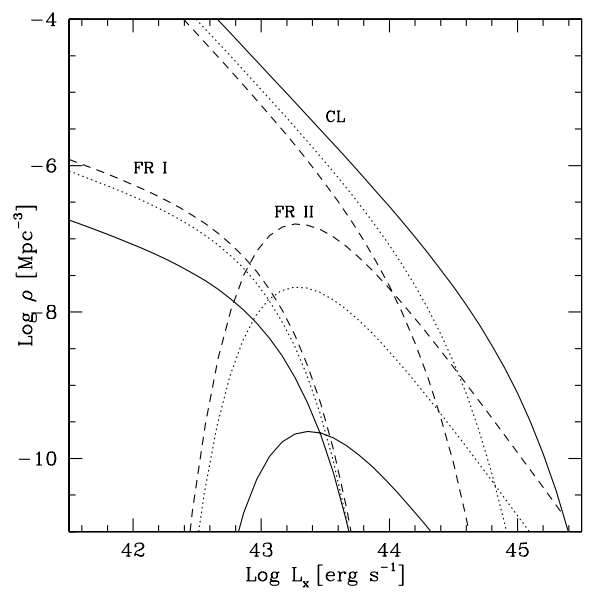

Figure 1. X-ray luminosity function of clusters (CL) and radio galaxies adapted from the $151 \mathrm{MHz}$ luminosity function (Willott et al 2001) assuming $\nu_{\mathrm{R}} L_{\mathrm{R}}=\nu_{\mathrm{X}} L_{\mathrm{X}}$ (Celotti \& Fabian 2004). Solid, dotted and dashed lines refer to $z=0,1$ and 2 .

\section{Cluster cores}

The radiative cooling time within the inner $100 \mathrm{kpc}$ of most cluster cores is less than $10^{10} \mathrm{yr}$. The gas temperature also drops there by a factor of about three (Fig. 2). If there is no heating of the gas it should cool out at a rate given by (see Fabian 1994 for a review)

$$
\dot{M}_{\mathrm{X}}=\frac{2}{5} \frac{L \mu m}{k T},
$$

reduced by a factor of about two by gravitational energy release as the gas flows. To maintain the pressure required to support the weight of overlying gas, the cooling gas flows inward, forming a cooling flow.
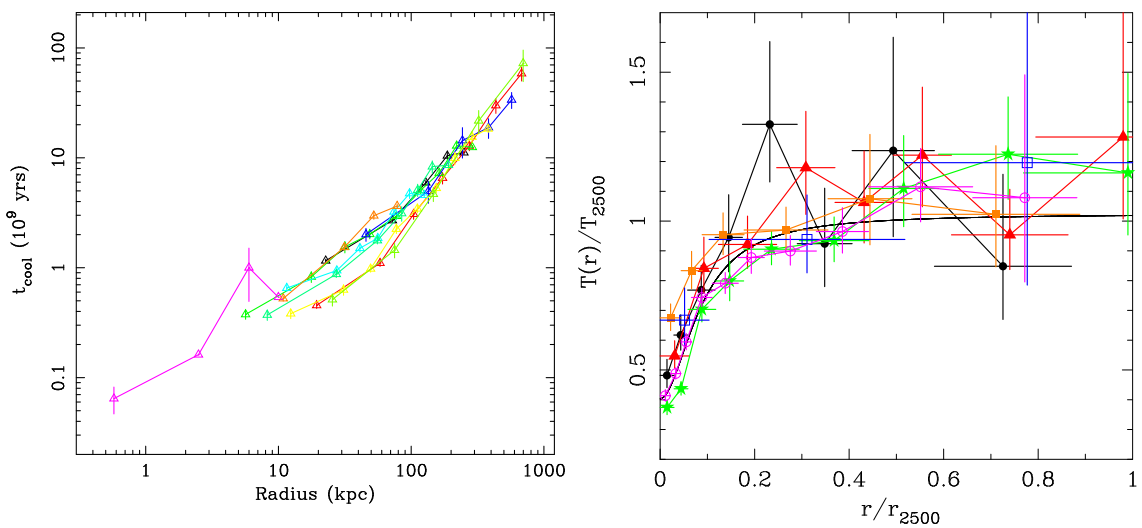

Figure 2. Left: Radiative cooling time versus radius for several cooling flow clusters (Voigt \& Fabian 2003). Right: Temperature profile for 6 massive clusters (Allen et al 2001). 


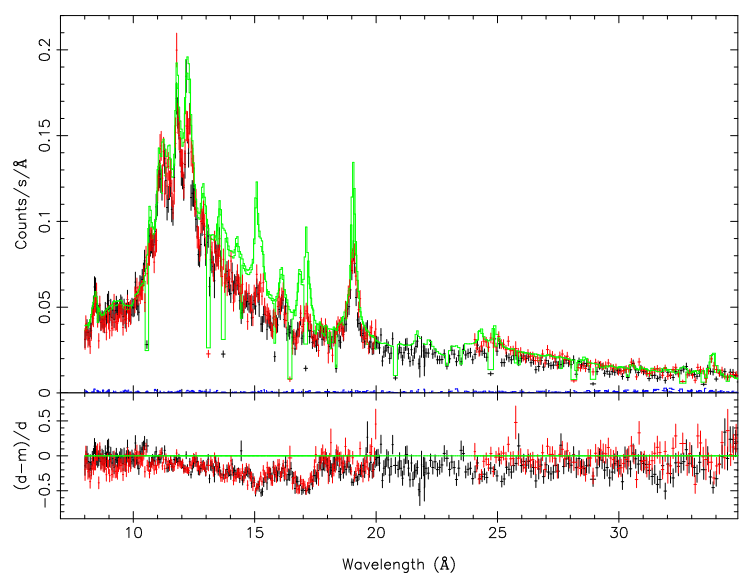

Figure 3. RGS X-ray spectrum of the inner parts of the Virgo cluster around M87 (Sakelliou et al 2002). The faint line shows the predicted emission if gas is cooling to below $10^{6} \mathrm{~K}$. The data clearly shows much less emission than predicted by this model in the 13-18 A region.

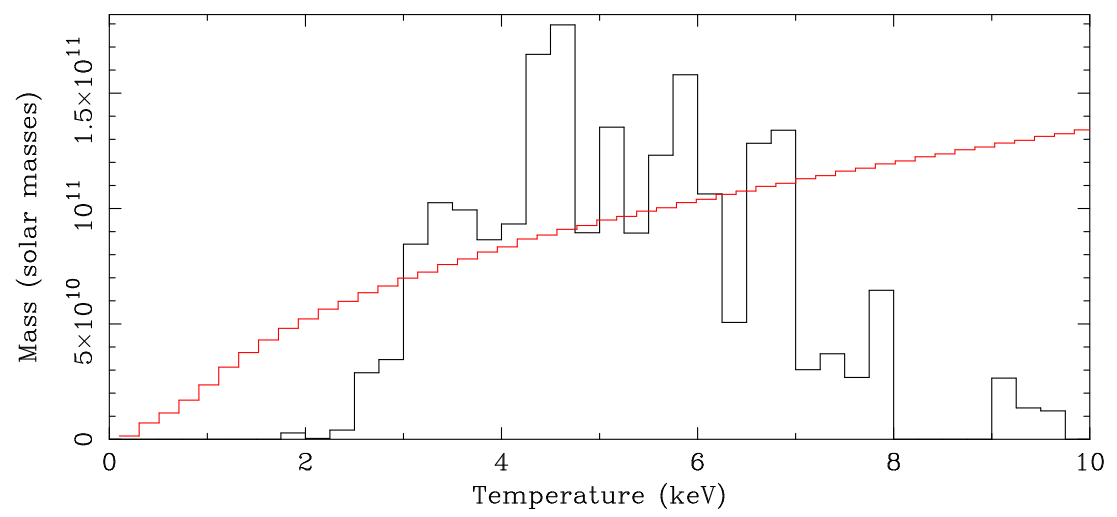

Figure 4. Mass - temperature distribution in the core of the Perseus cluster (Sanders et al 2004). Gas is seen down to about $2.5 \mathrm{keV}$. The curved line shows the expected mass distribution from gas cooling at constant pressure from the (outer) virial temperature $(7 \mathrm{keV}$ for Perseus) to below $0.1 \mathrm{keV}$. Gravitational work done as the gas flows, and isochoric cooling if magnetic pressure dominates, would reduce the low temperature prediction by a factor of up to 3 . Nevertheless no gas is seen below $1.5 \mathrm{keV}$.

As the gas cools below $1 \mathrm{keV}$ it emits strong Fe L line emission (e.g. FeXVII emission at 15 and $17 \mathrm{~A})$. A major result from the Reflection Grating Spectrometer (RGS) on XMM-Newton was to show that little such emission is seen (Peterson et al 2001, 2003; Tamura et al 2001; Kaastra et al 2004; Fig. 3). These studies show that the mass cooling rate below about one third of the bulk cluster temperature is less than one fifth to one tenth of that deduced from the above simple formula. Chandra data give the same result.

The effect was present in previous ASCA and ROSAT studies (e.g., Allen et al 2001) where the lack of emission had been attributed to intrinsic absorption. 
Although some absorption has not been completely ruled out, the improved new spectra show that it is either not dominant or intrinsic to the coolest gas.

The gas in a cluster core appears to cool by about a factor of three from the cluster virial temperature but no further. Since $t_{\text {cool }} \propto T^{2}$ at constant pressure around $1 \mathrm{keV}$, the cooler the gas the faster it cools. Why it does not cool further is puzzling and constitutes the 'cooling flow problem'. Just rearranging the cooling gas or only heating the coolest gas does not solve this problem. The data (Fig. 4) show no accumulation of gas at any particular temperature, just a lack of gas below about $1 / 3$ of the virial temperature.

It is likely that some heating is taking place, for which there are two plausible, long-known, candidates. They are heating by conduction from the hot outer gas and heating by a central active nucleus (Tucker \& Rosner 1983; Rosner \& Tucker 1989; Tabor \& Binney 1993; Binney \& Tabor 1995). However, a range of one-dimensional heating models investigated by Brighenti \& Mathews (2003) and by Ruszkowski \& Begelman (2002) shows that the solution of the cooling flow problem is not trivial.

\section{(a) Thermal conduction}

Thermal conduction has long been considered to be suppressed in clusters because of the observed central temperature drop. Conductive energy flow increases strongly with temperature, unlike radiative cooling which decreases (at constant pressure), so one might assume that it either operates, so making the core isothermal, or is suppressed and radiative cooling dominates. Narayan \& Medvedev (2001) have however revived the concept and noted that conduction may account for the observed temperature gradients. This has been explored in more detail by Fabian et al (2002b), Voigt et al (2002; Fig. 5) and Voigt \& Fabian (2004). These last authors found some clusters where conduction appears to be insufficient, therefore ruling out simple electron conduction models. A further issue is whether the effective conductivity can even be as high as the Spitzer value, or whether magnetic fields inferred from Faraday rotation (Fig. 6) suppress it heavily. (Note also that conduction models do not explain where the large observed temperature drop comes from in the first place.)

Cho et al (2003) have pointed out that turbulent heat diffusion may be important in cluster cores. Lisa Voigt and I (2004) have found that it is of the right form and magnitude $\left(\kappa_{\text {turb }}\right.$ in Fig. 5$)$ to explain the observations if the diffusion coefficient is about $0.2-0.5$ times the sounds speed times the radius (see also Kim \& Narayan 2003). Such a model assumes that large scale turbulent motions of gas enable efficient heat exchange within the core. Note that radial flows require energy, given the outward increasing entropy gradient, and moreover that abundance gradients (Fig. 6) must be maintained.

\section{(b) The central AGN}

All of the relevant cooling flow clusters peak on a central galaxy which is expected to host a massive black hole. Many of these galaxies have radio sources, some of which are obviously blowing bubbles of relativistic plasma in the central regions (e.g., Perseus, Fabian et al 2000, 2003, Fig. 7; A2052, Blanton et al 2001; A2597, McNamara et al 2001; A4059, Heinz et al 2002). The energy flux from the 


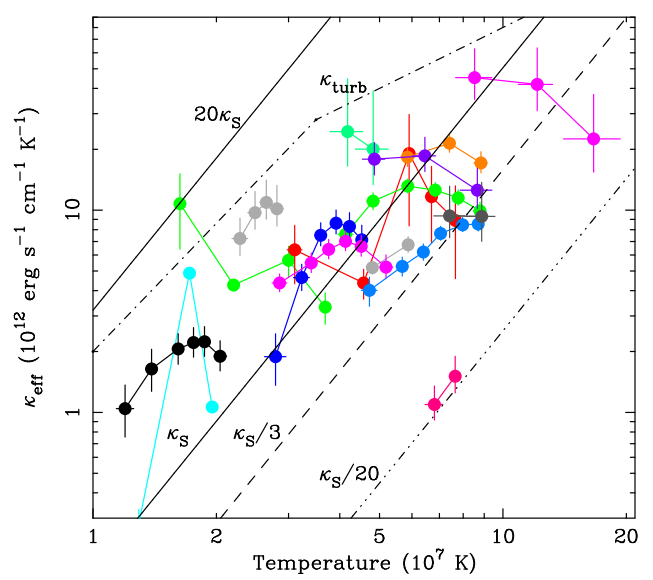

Figure 5. Effective conductivity required to balance radiative cooling in a sample of the brightest clusters (Voigt \& Fabian 2004). The Spitzer rate is labelled $\kappa_{\mathrm{S}}$.
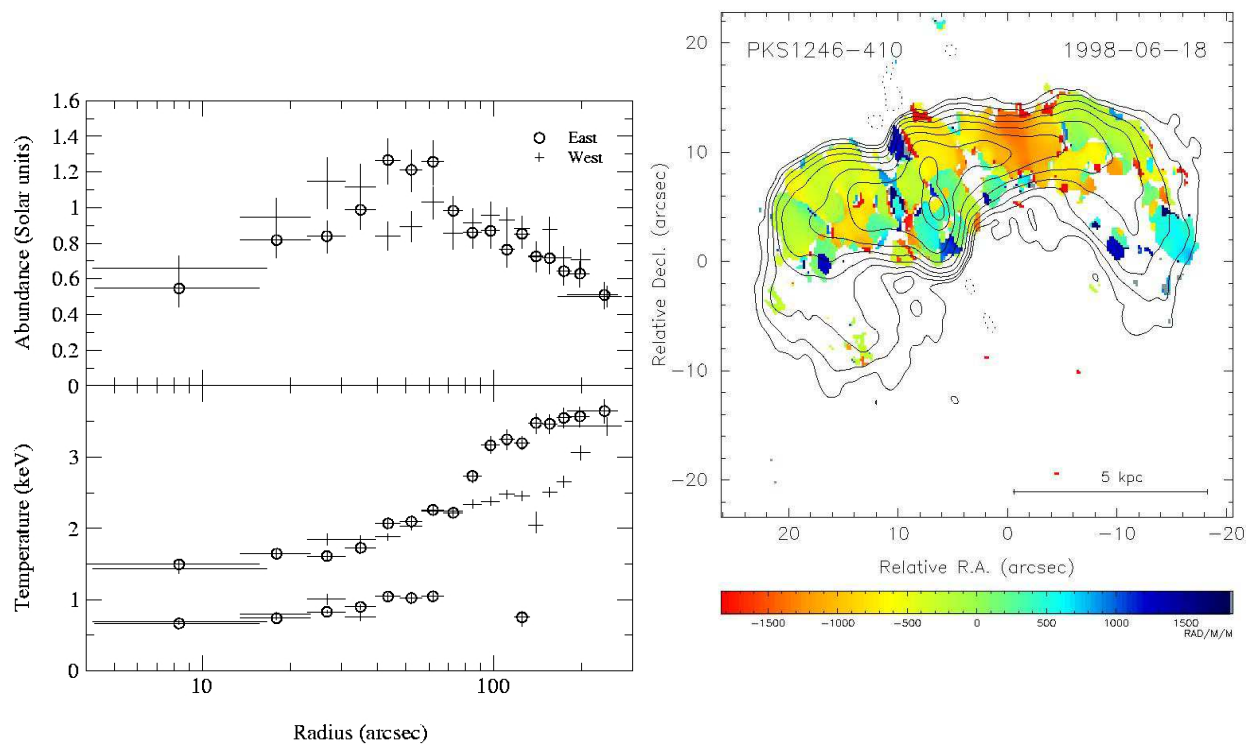

Figure 6. Left: Abundance (top) and temperature (bottom) profiles for the Centaurus cluster (Sanders \& Fabian 2002). Right: Faraday rotation measure map for the Centaurus cluster (Taylor et al 2002).

radio source can be high $\left(10^{43-45} \mathrm{erg} \mathrm{s}^{-1}\right)$; the jet power can be determined from the age and size of the bubbles (Fabian et al 2002).

The heat must be distributed (Johnstone et al 2002, Voigt \& Fabian 2003; Fig. 8) and cannot just heat the innermost, coolest gas (Voigt \& Fabian 2003). Some (Binney \& Tabor 1995; Kaiser \& Binney 2003) have argued that radio source activity may be sporadic, which explains why there is little correlation between the present 

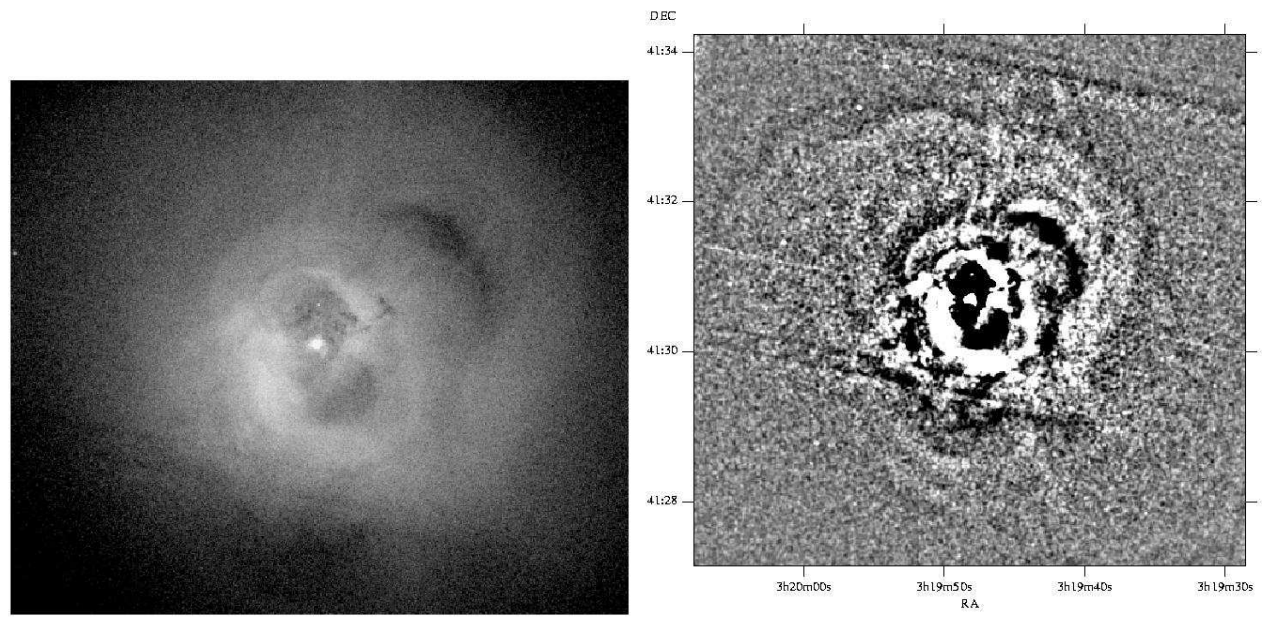

Figure 7. Left: X-ray image of the core of the Perseus cluster. Note the holes, which coincide with the radio lobes, above and below the nucleus. A buoyant outer bubble is seen to the right (Fabian et al 2003a). Right: Unsharp-masked X-ray image showing the ripples (Fabian et al 2003a).
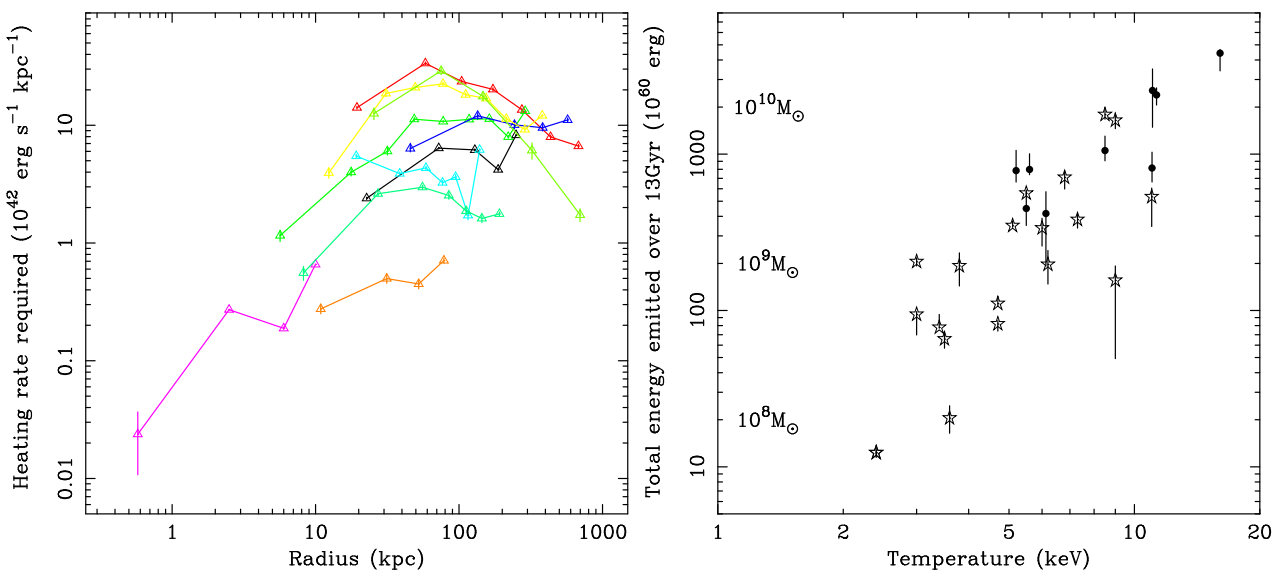

Figure 8. Left: The heating rate per unit radius required to stop radiative cooling in several cooling flow clusters (Voigt \& Fabian 2003). The heating needs to be distributed. Right: The total energy radiated over a Hubble time from within the cooling region for a selection of cooling flow clusters (Fabian et al 2002b). The masses indicate the total mass which must accrete to produce this energy if the efficiency of energy release is 0.1 ; the central black hole must therefore be more massive than this. If the central radio source stems cooling in the hotter clusters then most of the power released must be channeled into heating the intracluster medium.

radio source activity and the heating requirement. For a large duty cycle this makes very strong demands on the power of the source when it is switched on, particularly in the high luminosity clusters (Fig. 8), where it must exceed $10^{46} \mathrm{erg} \mathrm{s}^{-1}$. This will hardly be contained in simple bubbles, but will expand and dump its energy into the outer cluster.

Strong abundance gradients are found in many clusters (Fukazawa et al 2004), 
often peaking at radii $\sim 30 \mathrm{kpc}$ (Johnstone et al 2002; Fig. 6, Sanders \& Fabian 2002). This limits the degree of large scale disturbance taking place in the central regions. The apparent central drop in abundance is puzzling (if not due to resonance scattering).

Further difficulties are that the X-ray coldest observed gas lies around the bubbles, not all clusters host powerful enough radio sources. The mechanism for heat transfer from bubbles to the surrounding gas has been unclear, despite several impressive computational studies (e.g. Brüggen \& Kaiser 2002, Quilis et al 2002; Brüggen 2003; Basson \& Alexander 2003; Robinson et al 2003; Omma et al 2004) and analytical work (Churazov et al 2001; Soker et al 2003). None of the early simulations makes bubbles resembling those observed.

The creation of the bubbles or cavities means that $P d V$ work is done on the surrounding gas. This will create sound waves which can transport that energy away. The sound waves are indeed observed in our deep image of the Perseus cluster (Fig. 7; Fabian et al 2003a) as ripples in the X-ray surface brightness. The wavelength is about $10 \mathrm{kpc}$ which, given the sound speed of the gas, means a period of about $10^{7} \mathrm{yr}$. This agrees with the timescale on which bubbles would be formed and then buoyantly detach from a steady jet at the centre (Churazov et al 2001). Of course it does not need to be exactly steady but it could be so.

Interestingly we have found that the sound waves will dissipate their energy over the cooling region if the (ion) viscosity of the gas is close to the SpitzerBraginskii value for an unmagnetized plasma (Spitzer 1962). This would provide a splendid way for the energy produced by accretion onto the central black hole to be dissipated in a general, isostropic manner in the surrounding hot gas. Normally, energy from accretion does not couple well with hot gas since if electromagnetic the gas is transparent to the radiation and if kinetic the power is generally highly collimated.

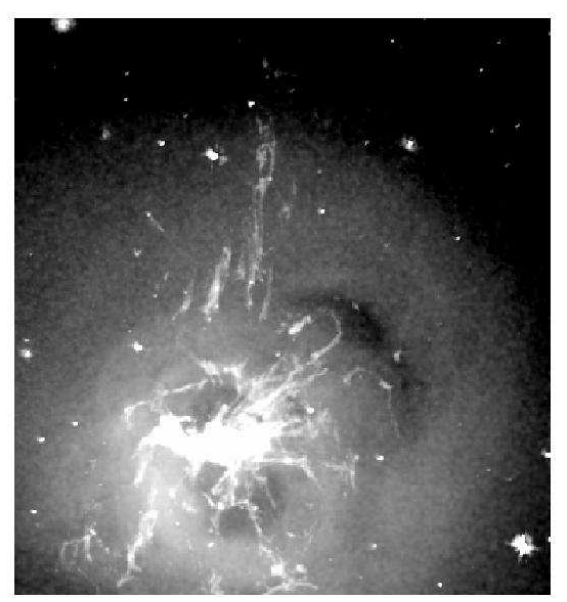

Figure 9. The $\mathrm{H} \alpha$ image (Conselice et al 2000) superimposed on the X-ray image of the centre of the Perseus cluster (Fabian et al 2003b). Note the many long straight outer filaments and the horseshoe ones inward of the outer bubble. 


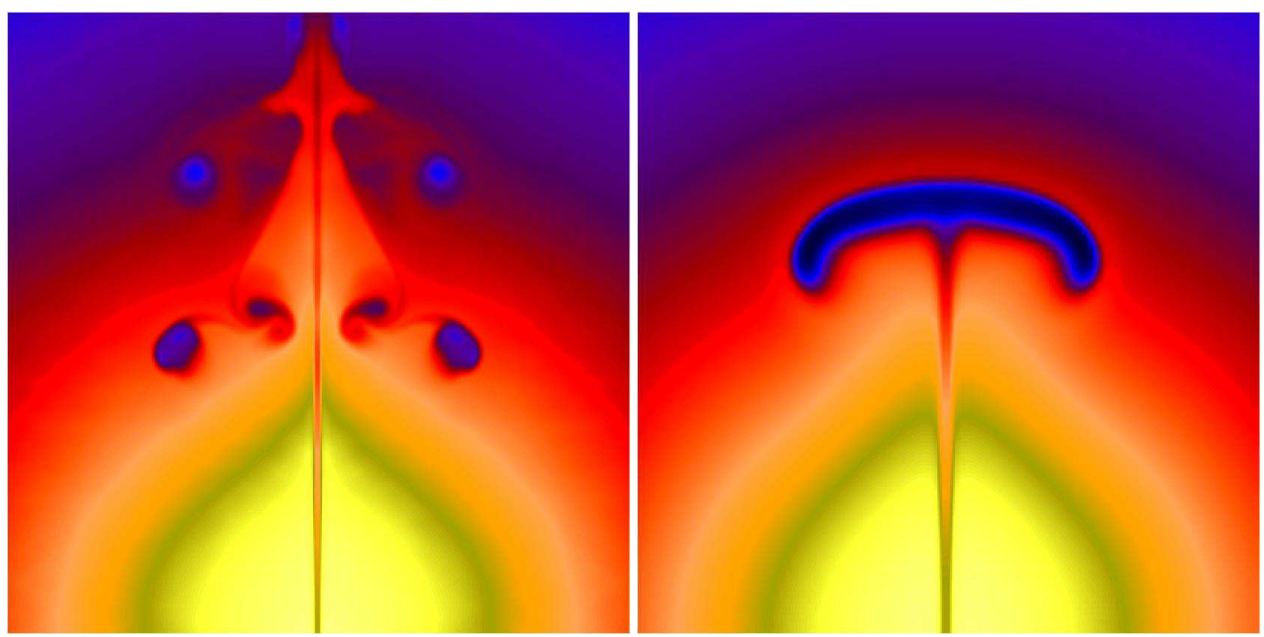

Figure 10. Gas density after a bubble has been released (left) in gas with no viscosity and (right) when the viscosity is one half the Spitzer value (from Reynolds et al 2004). Note that only in the viscous case does an intact outer bubble, such as observed in the Perseus cluster (Fig. 9) occur.

\section{(c) The viscosity of the ICM}

We have some independent evidence that the viscosity is high from the $\mathrm{H} \alpha$ emitting optical filaments seen (e.g. Conselice et al 2000) in the Perseus core around the central galaxy NGC 1275. If the viscosity is low then the hot gas should be turbulent, yet the filaments, which are less than a parcsec thick as estimated from their brightness, are often very straight for tens of kpc (Fig. 9). Moreover, just within the outer bubble there are two 'horseshoe-shaped' filaments which reveal a laminar flow pattern in the gas. By analogy with studies of rising cap bubbles in water, we assume that the Reynolds number of the flow is 1000 or less and thus deduce that the viscosity is high, with a factor of ten of the Spitzer-Braginskii value (Fabian et al 2003b). I conclude that the gas here is not turbulent, at least on scales less than $50 \mathrm{kpc}$.

Studies of flows within the ICM will be opened up by ASTRO-E2 which is due to launch next February (2005). Its $7 \mathrm{eV}$ spectral resolution will enable velocities to be determined from $\mathrm{Fe} \mathrm{K} \alpha$ lines to a few $10 \mathrm{sm} \mathrm{s}^{-1}$.

Do the likely tangled magnetic fields in the hot gas mean that the viscosity is much reduced? In accretion discs, the shear in the plasma increases the viscosity by many orders of magnitude. The situation for the intracluster medium is unclear but note that Faraday Rotation studies suggest the field is organised within cells of a few kpc so approximating a simple field structure on scales comparable to the ion mean free path. This may mean that the ion viscosity is close to the SpitzerBraginskii value. Unlike electron conduction, where the heat flow can be impeded by mirroring of particles or by an interface of magnetic structures, sound energy flows approximately isotropically through such barriers.

Simulations are only just beginning to include viscous effects. Ruszkowski \& Begelman (2003) and Reynolds et al (2004) have shown that it can have an important effect on ICM heating due to bubbles. In particular, the latter study shows 
that only with significant viscosity do whole detached bubbles occur (Fig. 10). A viscous medium can also tap energy from waves generated by subcluster mergers as well as by a central radio source.

\section{(d) General comments}

It is unlikely that either radio source heating or conduction can suppress radiative cooling within such a large region completely. Indeed, this is unnecessary, for significant rates of massive star formation (Crawford et al 1999) and masses of cold gas (Edge 2001) are found in the central parts. It is probable that

$$
\dot{M}_{\text {cool }}=\dot{M}_{\mathrm{X}} / 10
$$

where $\dot{M}_{\mathrm{X}}$ is the simple cooling rate derived simply from the X-ray data on the assumption of no heating. (It is simple to reduce the rate in equation 3.1 by a factor of 2-3 by including the gravitational heating and compression of magnetic field in the flow - the difficulty lies in reducing it by a further factor of 2-3.) The mass cooling rates previously inferred to be in the range $100-1000 \mathrm{M}_{\odot} \mathrm{yr}^{-1}$ spread over $100 \mathrm{kpc}$ are more likely to be $10-100 \mathrm{M}_{\odot} \mathrm{yr}^{-1}$ concentrated within the inner $30 \mathrm{kpc}$. The residual X-ray spectral mass cooling rates are then consistent with the observed star formation (e.g. RX J0820.9+0752, Bayer-Kim et al 2002; A1068, McNamara, Wise \& Murray 2004; both objects have central radio sources which

are currently weak). Some form of feedback presumably operates to keep $\dot{M}_{\text {cool }}$ at a low value.

In summary there are plausible heat sources at the centre and beyond radii of $100 \mathrm{kpc}$. The main problem is to distribute the energy within $100 \mathrm{kpc}$ without either disrupting the metallicity profiles or exceeding some observational constraint. Beyond any bubbles and plumes, and an occasional cold front (all of which occupy only a small fraction of the volume of the cooler gas) the distribution of surface brightness, temperature, metallicity and entropy of the gas all vary very smoothly. Gentle transport processes in the (magnetized) intracluster gas seem to be crucial. Improvement of our understanding of these processes is vital for further progress.

Further possibilities remain in which cooling dominates but the situation is more complicated so that gas cooling below say $2 \mathrm{keV}$ is less observable. This can result if the metals are not uniformly mixed in the hot gas (Morris \& Fabian 2003) or if the cooler gas mixes with cold gas (Fabian et al 2002). The missing soft Xray luminosity from a simple cooling flow is similar to that in the optical/UV/IR nebulosity at the centre. One reason to continue to consider such models is the detection of strong OVI emission in some clusters (Oegerle et al 2001).

The cooling flow problem, as it has become known, has wider relevance than just to cluster cores. It is also seen in the central interstellar medium of elliptical galaxiesm meaning that the problem occurs over a factor $10^{5}$ in luminosity! Moreover, galaxies grow as gas cools in their dark matter potential wells (White \& Rees 1978, Kauffmann et al 1999) and the cores of clusters are a directly observable example of this process. If it does not operate in cluster cores why does it work in galaxies? It is possible that whatever is stemming cooling in clusters is less effective in galaxies, operating in such a way as to dominate only in massive systems. It therefore determines the upper mass limit of visible galaxies (Binney 2004). Processes like conduction (Fabian et al 2002b), or viscosity, which are more effective in hotter, 


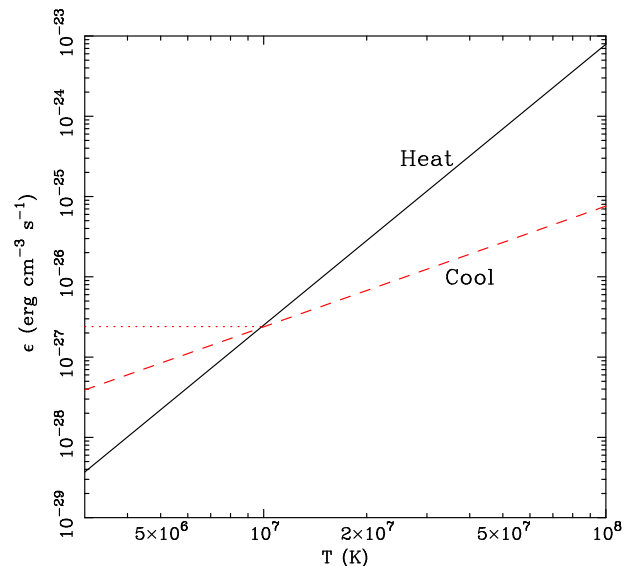

Figure 11. Viscous heating rate compared with the cooling rate for an atmosphere of temperature $T$. Note that heating wins above about $1 \mathrm{keV}$ and cooling below. Sound waves with a wavelength of $10 \mathrm{kpc}$ and high amplitude have been assumed.

massive objects, have the right property to allow gas to cool in normal galaxies but not in more massive systems (Fig. 11).

Both turbulent conduction and viscosity are highly temperature dependent. Kinetic energy fed into the core either by turbulence or sound waves, generated either from the outer parts of the cluster by subcluster mergers or from the centre by the central active nucleus, will dissipate and heat the inner regions, so offsetting the slower radiative cooling of gas above $10^{7} \mathrm{~K}$. Perhaps the crucial ingredient is that a massive galaxy has the extensive dense hot atmosphere at the centre of a group or cluster.

\section{Final comments}

AGN can have, and probably did have, a profound effect on the ICM. The radio luminosity function (Fig. 1) shows that FRII radio sources dominated the energy budget at redshifts of $1-3$ whereas FRI sources dominate now. FRII sources acting within the subclusters which have now assembled into present-day clusters would have seriously heated and disturbed the ICM there, and could have injected the heat needed to make $L_{\mathrm{X}} \propto T^{3}$. It is likely that FRI sources in central cluster galaxies at the present epoch continue to heat the innermost $100 \mathrm{kpc}$ or so of the ICM gently, preventing central galaxies from accreting much more gas and determining their final stellar mass.

\section{Acknowledgments}

This is an expanded and updated version of an earlier review. I thank the Royal Society for support. 


\section{References}

Allen S.W., Fabian A.C., Johnstone R.M., Arnaud K.A., Nulsen P.E.J., 2001, MNRAS, 322, 589

Allen, S.W., Schmidt, R.W., Fabian, A.C. 2001, MNRAS, 328, L37

Basson J.F., Alexander P., 2003, MNRAS, 339, 353

Bauer, F.E., 2002, AJ, 123, 1163

Bayer-Kim C.M., Crawford C.S., Allen S.W., Edge A.C., Fabian A.C., 2002, MNRAS, 337, 938

Binney J., 2004, MNRAS, 347, 1093

Binney J., Tabor G., 1995, MNRAS, 276, 663

Blanton E.L., Sarazin C.L., McNamara B.R., Wise M.W., 2001, ApJ, 558, L15

Brighenti F., Mathews W.G., 2003, ApJ, 587, 580

Brüggen M., Kaiser C.R., 2002, Nature, 418, 301

Carilli, C.L., Harris, D.E., Pentericci, L., Röttgering, H.J.A., Miley, G.K., Kurk, J.D., van Breugel, W., 2002, ApJ, 567, 781

Celotti A., Fabian A.C., 2004, MNRAS, in press (astro-ph/0405622)

Cho J., Lazarian A., Honein A., Knaepen B., Kassinos S., Moin P., 2003, ApJ, 589, L77

Churazov E., Brüggen M., Kaiser C.R., Böhringer H., Forman W., 2001, ApJ, 554, 261

Churazov E., Sunyaev R., Forman W., Bhringer H., 2002, MNRAS, 332, 729

Conselice, C.J., Gallagher J.S., Wyse R.F.G., 2000, ApJ, 559, 791

Crawford C.S., Allen S.W., Ebeling H., Edge A.C., Fabian A.C., 1999, MNRAS, 306,875

Edge A. C., 2001, MNRAS, 328, 762

Evrard A.E. et al., 2002, ApJ, 573, 7

Fabian A.C., 1994, ARAA, 32, 277

Fabian A. C. et al., 2000, MNRAS, 318, L65

Fabian A.C., Mushotzky R.F., Nulsen P.E.J., Peterson J., 2001, MNRAS, 321, L20

Fabian A.C., Allen, S.W., Crawford, C.S., Johnstone R.M., Morris, R.G., Sanders J.S., Schmidt R.W., 2002a, MNRAS, 332, L50

Fabian A.C., Voigt L., Morris R.G., 2002b, MNRAS, 335, L71

Article submitted to Royal Society 
Fabian, A.C., Celotti, A., Blundell, K.M., Kassim, N.E., Perley, R.A., 2002c, MNRAS, 331, 369

Fabian A.C., et al 2003a, MNRAS, 344, L43

Fabian A.C. et al 2003b, MNRAS, 344, L48

Fabian, A.C., Sanders, J.S., Crawford C.S., Ettori, S., 2003c, MNRAS, 341, 729

Fabian, A.C., Celotti, A., Johnstone, R.M., 2003, MNRAS, 338, L7

Fukazawa Y. et al., 1994, PASJ, 46, L55

Gastaldello F., Molendi S., 2004, ApJ, 600, 670

Heinz S., Choi, Y., Reynolds, C.S., Begelman M.C., 2002, ApJ, 569, L79

Inoue, S., Sasaki, S., 2001, ApJ, 562, 618

Johnstone R. M., Allen S.W., Fabian A. C., Sanders J.S., 2002, MNRAS, 336, 299

Kaastra, J., et al, 2004, A\&A, 413, 415

Kaiser C.R., Binney, J., 2003, MNRAS, 338, 837

Kauffmann G., Colberg J.M., Diaferio A., White S.D.M. 1999, MNRAS, 303,188

Kim W-T., Narayan R., 2003, MNRAS, 596, L139

McNamara B.R., et al 2001, ApJ, 562, L149

McNamara B.R., Wise, M.W., Murray S.S., 2004, ApJ, 601, 173

Morris R.G., Fabian A.C., 2003, MNRAS, 338, 824

Narayan R., Medvedev M. V., 2001, ApJ, 562, L129

Oegerle W.R., et al 2001, ApJ, 560, 187

Omma, H., Binney, J., Bryan G., Slyz, A., 2004, MNRAS, 348, 1105

Peterson J. R. et al., 2001, A\&A, 365, L104

Peterson J.A. et al, 2003, ApJ, 590, 207

Quilis V., Bower R.G., Balogh M.G., 2001, MNRAS, 328, 1091

Reynolds, C.S., McKernan, B., Fabian, A.C., Stone, J.M., Vernaleo, J.C., 2004, ApJ, in press (astro-ph/0402632)

Robinson, K. et al., 2004, ApJ, 601, 621

Rosner R., Tucker W.H., 1989, ApJ, 338, 761

Ruszkowski M., Begelman M.C., 2002, ApJ, 581, 223

Ruszkowski M., Begelman M.C., 2004, ApJ, in press (astro-ph/0310760) 
Sakelliou, I., et al 2002, A\&A, 391, 903

Sanders J. S., Fabian A. C., 2002, MNRAS, 331, 273

Sanders J. S., Fabian A. C., 2004, MNRAS,

Scharf, C., Smail, I., Ivison, R., Bower, R., van Breugel, W., Reuland, M., 2003, ApJ, 596, 105

Schmidt R.W., Allen S.W., Fabian A.C., 2001, MNRAS, 327, 105

Siemiginowska, A., Smith, R.K., Aldcroft, T.L., Schwartz, D.A., Paerels, F., Petric, A.O., 2003, ApJ, 598, L15

Soker, N., Blanton E.L., Sarazin C.L., 2002, ApJ, 573, 533

Spitzer L., 1962, Physics of Fully Ionized Gases, New York: Wiley-Interscience

Tabor G., Binney, J., 1993, MNRAS, 263, 323

Tamura T. et al., 2001, A\&A, 365, L87

Taylor G.B., Fabian A.C., Allen S.W., 2002, MNRAS, 334, 769

Valageas, P., Silk, J., 1999, A\&A, 350, 725

Tucker W.H., Rosner R., 1983, ApJ, 267, 547

Voigt L. M., Schmidt R. W., Fabian A. C., Allen S. W., Johnstone R. M., 2002, MNRAS, 335, L7

Voigt L. M., Fabian A. C., 2004, MNRAS, 347, 1130

White S.D.M., Rees M.J., 1978, MNRAS, 183, 341

Willott, C.J., Rawlings. S., Blundell, K.M., Lacy, M., Eales, S.A., 2001, MNRAS, 322,536

Wu, K.K.S., Fabian, A.C., Nulsen, P.E.J., 2000, MNRAS, 318, 889

Yuan, W., Fabian, A.C., Celotti, A., Jonker, P.G., 2003, MNRAS, 346, L7

Zakamska N.L., Narayan R., 2003, ApJ, 582, 162 